\title{
Coping with low modulation in speckle interferometry: a novel approach based on the Delaunay triangulation
}

\author{
Sébastien Equis and Pierre Jacquot \\ Nanophotonics and Metrology Laboratory, Swiss Federal Institute of Technology Lausanne, EPFL- \\ STI-NAM, Station11, CH-1015 Lausanne, Switzerland
}

\begin{abstract}
Interferometric signals involving speckle waves invariably exhibit phase indeterminations. These indeterminations arise at the zero-intensities of the speckle fields, or singularities, and show themselves as a net loss of modulation depth of the interferometric signals. To bypass the difficulty associated with the processing of low modulated parts of speckle interferometry signals, we propose a novel approach based on the Delaunay triangulation (DT). The method applies in both situations of static and dynamic regimes, and is designated respectively by "sine-cosine DT filter" and "3D piecewise processing" or 3DPP - 3D denoting the temporal and the two spatial coordinates of the recording. The task consists in discarding purely and simply the under-modulated parts of the signal according to a user-defined binary criterion, and filling the missing parts by interpolation. This first step provides a grid with nodes randomly occupied by reliable phase values or empty. At the empty nodes, the computed phase values result from a DT ensuring that the interpolation relies on the three closest well-behaved neighbors, followed by spline-fitting a smooth surface over them. In a dynamic regime - where the benefits of the temporal approach are unanimously acknowledged - the empirical mode decomposition is used to select the valid intervals and the Hilbert transform to compute phase data therein. We give a detailed description of the DT filtering techniques, show their ability to offer the optimal compromise between spatial and measurement resolutions depending on the user-chosen binary criterion and highlight some definite advantages over classical filtering methods in terms of phase error reduction and algorithmic complexity.
\end{abstract}

Keywords: speckle interferometry, phase extraction, low modulation, singularities, piecewise processing, Delaunay triangulation, empirical mode decomposition, Hilbert transform.

\section{INTRODUCTION}

The many diverse speckle interferometry (SI) techniques have gained a wide acceptance in applicative circles partly and certainly because simple models have been developed to satisfactorily explain their modi operandi. The increasing use of SI in photomechanics, for instance, gives an indication of the scope of this remark. Experimental mechanics scientists are confident to dispose through SI techniques of a set of measurement methods leading to accurate and highly resolved micro-displacement and deformation fields, based on a comprehensible and well established body of theories. Not surprisingly, the simple two beam interference formula is at the core of the theoretical background, with the recognition that the three constituents of the resultant intensity - i.e. the background, the modulation and the phase - are now random variables, in opposition to classical interferometry. In SI, the resultant intensity, or more strictly its recording, is dubbed "specklegram". Next enters the assumption of the invariance of the three above quantities under a small perturbation of the interferometric system, the phase being only deterministically incremented in direct proportion of the perturbation. Then it is easy to generate the so-called correlation fringes by subtracting two specklegrams associated with two slightly different interference states, visualizing the evolution of the investigated phenomenon. Quantitatively, many techniques are available to extract digitally the deterministic phase in question. To loop the loop, establishing the relationship between the phase increment and the perturbation can be done by straightforward first order approximations of the optical path change involved in that perturbation. This last issue is in no way considered in this article, where we will concentrate on the sole problem of the effects of the random nature of the modulation.

Now, if the statistical properties of the modulation are closely taken into account, in particular its definite probability to drop to zero, the above evoked model does not hold anymore. One should even realize that it is only by a bit of luck and generally in an average sense that it can work. A closer look is needed in order to provide the end users with a more reliable characterization of the performances of SI. Indeed, zero or low modulation is synonymous with zero or low

Speckle 2010: Optical Metrology, edited by Armando Albertazzi Goncalves Jr., Guillermo H. Kaufmann, Proc. of SPIE Vol. 7387, 738709 · (c) 2010 SPIE · CCC code: 0277-786X/10/\$18 · doi: 10.1117/12.868466

Proc. of SPIE Vol. 7387 738709-1 
intensity of at least one of the two interfering light field in the considered areas - a not exceptional situation with speckle waves, as will be first reminded below. In these cases, the phase of the specklegram is simply indeterminate. Any visualization technique and phase computation algorithm will fail to produce a correct solution in these regions. So far, all the established solutions to this well-known problem rely on the use of more or less sophisticated filtering procedures and apply mostly to phase maps: the grey level of each pixel is replaced by a certain combination of the grey levels of surrounding pixels according to ad hoc rules. These filtering procedures are briefly reviewed.

We thought it worth to revisit the subject and we develop here a new way to cope with the regions of zero or low modulation of the SI signals: the piecewise processing ${ }^{1,2}$. Signals intervals below a predefined modulation threshold are purely and simply discarded. The missing parts are rebuilt by an appropriate interpolation procedure using only the most reliable and closest preserved data, namely an interpolation based on the Delaunay triangulation (DT). Any routine spatial integration regime - weak, moderate or strong - is included in the study. We propose a solution for both the static and dynamic regimes of object deformation. Simulations as well as experimental results show the benefit of the piecewise processing procedure as compared to standard filtering approaches.

\section{SINGULARITIES AND INTERVALS OF LOW MODULATION IN SI SIGNALS}

In the complex plane, the tip of the vector $\boldsymbol{A}(s)$ representing the amplitude, $A$, and the phase $\psi$ of a speckle field follows a random path, $C(s)$, as shown in Fig. 1a). The parameter $s$ can be either the time or the curvilinear abscissa of a point along an arc in the plane where the speckle is observed. Aside from the current vector labeled by (1), the three particular realizations labeled (0), (2) and (3) are of special interest: (0) corresponds to a local intensity maximum, where the phase gradient corresponding to an increment of $s$ cannot be zero and the speckle phase $\psi$ is necessarily non stationary; in the region (2), on the contrary, the speckle phase does not change significantly: the region is a phase saddle $^{3}$; the worst case is when the curve $C(s)$ crosses the origin: the intensity of the field is zero and its phase is indeterminate.

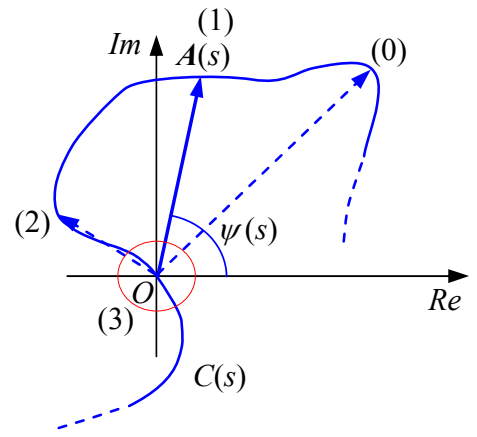

a)

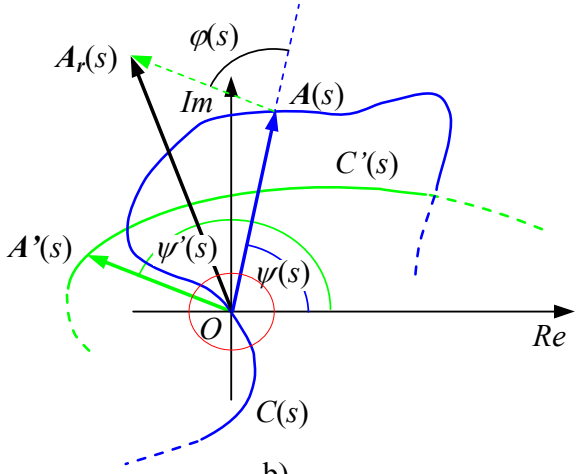

b)

Figure 1: a) Random evolution (spatial or temporal) of the complex amplitude of a speckle field; the four vectors labeled (0) to (3) represent a local maximum, an arbitrary behavior, a phase saddle and a singularity, respectively. b) Interferometry with at least one speckle wave; the interferometric phase $\varphi$ is indeterminate when the speckle component goes to zero.

In an interferometric experiment, two fields are added, Fig. 1b), and the recoverable quantity from an intensity measurement, $I$, is the angle $\varphi$ between the two components, as shown by both the sketch and the two beam interference formula:

$$
I(s)=I_{B}(s)+I_{M}(s) \cos \varphi(s) ; \quad I_{B}(s)=A^{2}(s)+A^{\prime 2}(s) ; \quad I_{M}(s)=2 A(s) A^{\prime}(s) ; \quad I_{M}(s) \leq I_{B}(s) .
$$

$I_{B}$ and $I_{M}$ are respectively the background (local average) and the modulation (half the local peak-valley) of the signal, and $\varphi$, as usual, will be simply named the phase. As soon as at least one of the interfering waves is a speckle wave, the three quantities $I_{B}, I_{M}$ and $\varphi$ are random variables. Eq. (1) stands for the aerial resultant intensity at a single spatiotemporal point. It turns out that the equation is formally the same in the case of spatial integration, as done by any pixelized detector. The integrated resultant intensity can be built as the sum of $m$ independent contributions. The definition of the number $m$ will be clarified below. The integrated background is equal to the sum of the $m$ backgrounds of the contributions. It is convenient to define a complex integrated modulation as the vectorial sum of the $m$ 
modulations of each contribution $k$ of type $\left(I_{M k} \cos \varphi_{k}, I_{M k} \sin \varphi_{k}\right)$. The resulting integrated modulation itself is the modulus of this vectorial addition in the complex plane and the integrated phase is its argument. Thus, in a spatially integrated regime, the singularities are the pixels exhibiting a complex modulation equal to zero.

When the speckle component enters the circle close to the origin, Fig. 1, the phase $\varphi$ clearly becomes indeterminate too, making completely meaningless the interference signal. Any standard phase extraction algorithm will fail to produce a correct solution. Hence, ideally, SI techniques should be restricted to the regions of phase saddles, mimicking the classical interferometry situation. This would however considerably reduce the number of measured points and, as known from a long time, reliable information can also be obtained outside the phase saddles ${ }^{4}$. Conversely, it could be tempting to favor pixels with the highest modulations, corresponding most often to the $(0)$ regions of local intensity maxima. Again this would reduce still more the number of measured points, since maxima are outnumbered by saddles and singularities in a ratio of 1 to $14^{3}$ and a trade-off has to be found between the density and the reliability of the phase measurements. Yet, in any case, it makes sense to consider the modulation depth of the SI signal as the primary parameter to be taken into account in the search for the best compromise.

Zero modulation - and the subsequent impossibility to compute the phase - occurs when at least one of the field components is zero, or amounts to small values if we want also to account for threshold and intensity resolution effects of the detector. In spatially integrated regimes, we have just seen that this situation occurs when the complex modulation is null. In these circumstances, noise is predominant in the interferometric signal. This state of affairs is worrying because not rare: e.g., in a classical spatially resolved Gaussian speckle field the zero intensity has the highest probability, and the singularities are as many as speckle grains ${ }^{5}$. Furthermore, in order to better estimate the probability of occurrence of these events, we should have a closer look to the probability density function of the modulation, $P_{I M}\left(I_{M}\right)$. This density is likely to take different forms, related to four possible cases: the interference of $i$ ) two speckle fields or ii) one speckle- and a smooth field, the iii) spatially resolved and iv) un-revolved regimes. The four forms are known, either as an explicit analytic expression or by numerical integration of the joint probability distribution of the background and modulation ${ }^{6-7}$. Fortunately, if we consider the most usual SI recording conditions, the four forms can be reduced to a single one with a very good approximation, namely the Rayleigh distribution:

$$
P_{I M}\left(I_{M}\right)=\frac{m I_{M}}{2\left\langle I_{C}\right\rangle^{2}} \exp -\frac{m I_{M}^{2}}{4\left\langle I_{C}\right\rangle^{2}} .
$$

In order to simplify this expression without changing its significance, we chose the same average intensity $\left\langle I_{C}\right\rangle$ for the two field components. The approximation is found very good ${ }^{6-7}$ provided that $m$ is kept greater than 2.5. The parameter $m$ indicates the degree of spatial integration at the recording of the specklegram ${ }^{8-9}$. It can be understood as the number of independent cells per pixel. By construction, the cells are all of the same size, small enough so that the speckle intensity is constant within each cell, and large enough to ensure the statistical independence of the integrated intensity from cell to cell. This implies that the cells are always smaller than the speckle size, reaching asymptotically the same values only for very large $m$ (e.g.: $m>100$ ). On the opposite side, $m$ tending toward 1 means about one cell per pixel, typical of a spatially fully resolved regime. However, in practice, such regimes are never used, since they suppose imaging systems of extremely low apertures (typically an aperture number greater than 100). The mathematical expression for $m$ is too involved ${ }^{9}$ to be given here. It is sufficient to remind that $m$ ranges from about 4 to 80 for the usual aperture numbers between 8 and 1.2, around a wavelength of $550 \mathrm{~nm}$, and for $6 \times 8 \mu \mathrm{m}^{2}$ pixels ${ }^{6}$. The Rayleigh probability density function of the modulation is plotted in Fig. 2a) for the three spatial integration regimes, weak, moderate and strong, i.e. for $m=4,16$ and 64 .

Though the probability density of the modulation is zero at the origin, it takes rapidly increasing values, especially when the spatial integration becomes larger and larger. This point is confirmed by the shape of the curve representing the probability $\mathcal{P}$ to find a low modulation, Fig. $2 \mathrm{~b}$ ). This figure plots the probability to obtain a modulation smaller than an arbitrary small value $\alpha\left\langle I_{C}\right\rangle$ :

$$
\mathrm{P}\left(I_{M} \leq \alpha\left\langle I_{C}\right\rangle\right)=1-\exp -\frac{m \alpha^{2}}{4} ; \alpha \geq 0 \text {. }
$$

It can be seen that a probability as high as up to $40 \%$ can occur for a range of poor modulations, namely the modulations smaller than $0.2\left\langle I_{C}\right\rangle$. The important conclusion to be drawn is the following: for any types of SI arrangements, 
involving only one or two speckle waves, operated in normal spatial integration regimes, i.e. in the usual range of apertures and pixel areas, it is unfortunately expected that one has necessarily to cope with low, unexploitable and not infrequent modulations.

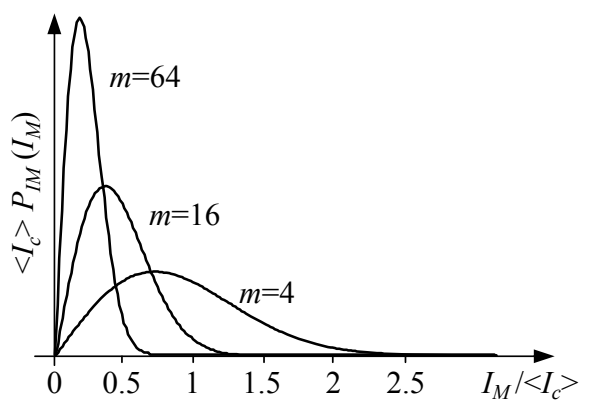

a)

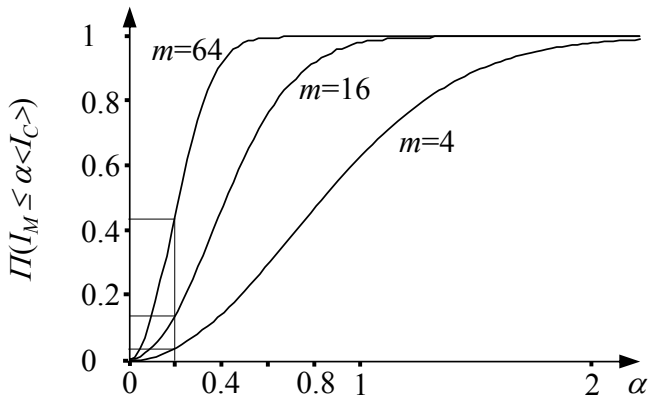

b)

Figure 2: a) Rayleigh distribution of the modulation $I_{M}$ for three degrees of spatial integration, $m=4,16,64$, representing the cases of two speckle waves or one speckle and one smooth wave interferences. b) The probability $\mathcal{P}$ of the modulation to be under a predefined threshold.

Another incentive to be very cautious with respect to the low modulated parts of SI signals is that the phase error due to pupil- and image-plane decorrelation is, incidentally, to a good approximation, inversely proportional to the pixel modulation ${ }^{7,10}$, speaking here again for special phase extraction treatments in such low modulated regions. The most usual of these treatments, and, by contrast, the DT techniques are presented in the forthcoming sections.

\section{FILTERING STRATEGIES}

As long as phase extraction procedures are concerned, it is convenient to distinguish between two main categories of deformation measurements: the static and dynamic regimes of loading ${ }^{11}$. In this context, static means that three conditions are fulfilled by the acquisition system: $i$ ) the exposure time is short enough, ii) the frame acquisition rate high enough; and iii) the phase-shifting device fast enough, so that several phase-shifted versions of the same state of deformation can be recorded. The choicest phase extraction methods are then precisely the well-known standard phaseshifting methods applied to both the initial and final states of the deformation. The phase map related to the deformation is the subtraction modulo $2 \pi$ of these two random phase maps. Of course, whatever the chosen $n$-image algorithm, the computed phase for the intervals of zero or nearly zero modulations is completely wrong. The best known and more widely accepted technique is then to filter out the deformation phase map by the so-called sine-cosine linear filter. In its more advanced version, the coefficients of the convolution kernel are proportional to the square of the pixel modulation ${ }^{7,10}$, for the reasons evoked at the end of the previous section. This filter not only preserves the sharpness of the $2 \pi$ phase jumps, essential for the next unwrapping operation, but, applied iteratively and with anisotropic kernels, it also maintains the loss of spatial resolution in tolerable proportions ${ }^{12}$. Though efficient, this procedure has nonetheless the effect of spreading the phase indetermination errors over the kernel area, not to mention its failure when the kernels happen to contain mostly invalid data. In section 5 , we shall see that the sine-cosine DT filter can favorably compete with the best phase maps filtering methods.

In dynamic loading conditions, two ways are open: $i$ ) to process spatially each of the $2 \mathrm{D}$ correlation fringe patterns obtained by subtracting two by two the acquired specklegrams according to a given scheme; ii) to analyze separately each 1D pixel signal extracted along the time axis. In both cases, many methods are available ${ }^{13}$. We will simply notice here that the most widespread method of the first category is the Fourier transform (FT) method combined with the introduction of a spatial carrier ${ }^{14}$. The filter is the inverse FT of the mask function selecting the appropriate part of the correlation fringe spectrum. It turns out that the phase extraction is highly filter-dependent and prone to border effects, resulting in the basic difficulty to evaluate correctly the measurement and spatial resolutions. Moreover, the FT method is fundamentally not well adapted to the analysis of non-stationary signals, where both the position in the pattern and the local spatial frequency are of primary interest. More recently, the pixel temporal analysis has gained appeal, especially with the introduction of the wavelet processing (Morlet wavelet: MW) ${ }^{15}$ and the joint use of the empirical mode decomposition (EMD) and Hilbert transform (HT) ${ }^{1,13}$. Even if MW and EMD-HT involve some intrinsic sort of filtering, creating a first data smoothing, they cannot escape the problem of the existence of low modulation intervals. 
They still call therefore for an additional post-filtering. In the case of dynamic SI, the 3D piecewise processing will thus be applied to the set of $1 \mathrm{D}$ temporal pixel signals.

\section{SINE-COSINE DT FILTER AND 3D PIECEWISE PROCESSING}

\subsection{Static regime}

The starting point is a wrapped phase map representing the $2 \pi$ phase difference between two static cases, obtained for instance with a phase-stepping algorithm. We have thus in hand a phase map in the range $[-\pi, \pi]$ with a pretty high amount of noise, which we want to remove as much as possible, whereas the sharpness of the $2 \pi$ jumps has to be preserved for the subsequent unwrapping step. The preservation of the spatial resolution is of course of primary importance as well. The widely adopted strategy is to apply filtering techniques on the cosine and the sine of the phase map and compute again the phase afterwards. The novel approach we bring here to tackle the modulation loss issue is to replace the classical filtering convolution by an interpolation procedure on the cosine and the sine of the phase separately. From the evident conclusion that the regions of low or null modulation can only produce random phase errors, the adopted strategy is to suppress purely and simply the pixels whose modulation has been estimated insufficient. Holes of random location and size are thus created within the map of phase difference and they must be filled by an interpolation procedure which relies on the remaining valid data. Given a set of points, randomly spread over a portion of space, a natural approach for recovering a smooth uniformly sampled surface, is to construct first a triangulation in the plane, then assign a weight to each point of the mesh and finally, build a piecewise polynomial surface over it. To preserve the spatial resolution at best, it makes sense to fill the gaps with the knowledge carried by the closest pixels. In other words, a desirable triangulation would minimize the size of the facets, and the Delaunay triangulation actually fulfils this criterion by maximizing the smallest angle of all the triangles of the mesh, avoiding this way skinny triangles. Once the triangulation has been computed with the Quickhull algorithm ${ }^{16}$, a cubic spline surface is applied over the tessellation (Clough-Tocher method) and the phase is eventually recomputed from the smoothed cosine and sine of the phase. The whole operation is illustrated in Fig. 3 by means of experimental signals obtained with a Michelson type speckle interferometer. The map of phase difference represents the out-of-plane displacement field of a diffusing metal plate held in three points on its periphery and loaded in its middle.

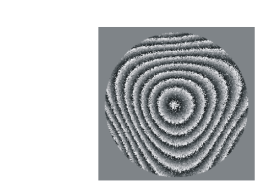

$\Delta \varphi$
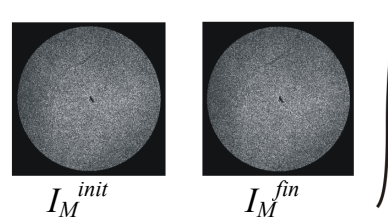

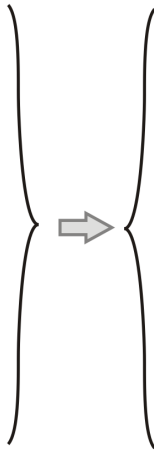

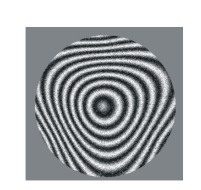
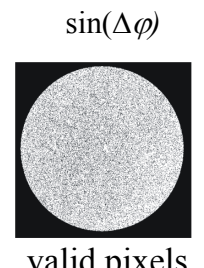
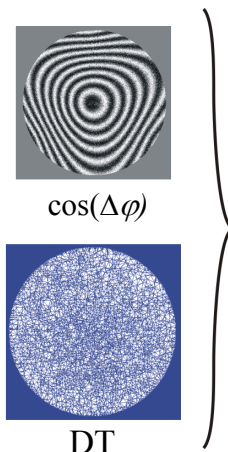

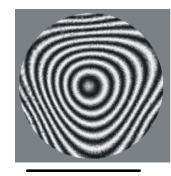

$\cos (\Delta \varphi)$

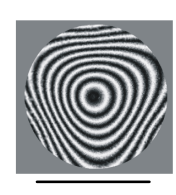

$\sin (\Delta \varphi)$

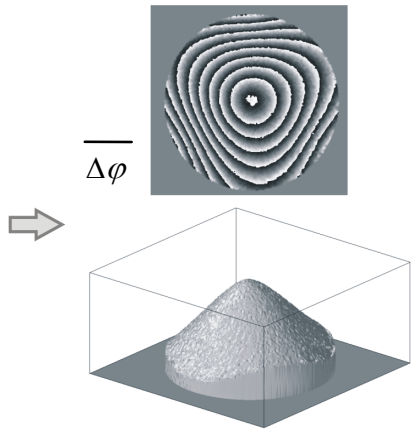

Figure 3: The input data are the phase difference map $(\Delta \varphi)$ and the intensity modulation maps for the initial and the final states (left); from those data, the map a) of valid pixels (modulation higher than a certain threshold) is computed and then b) the Delaunay triangulation (center); from the smoothed versions of the cosine and the sine of $\Delta \varphi$ (the top bar denotes the filtered version), the phase is retrieved (right).

Given a random set of points in the plane, the first step of the whole triangulation-interpolation process is to compute the convex hull of the data set in question. The convex hull in 2D might be seen as a rubber band surrounding a set of nails driven in a wooden board representing the mesh of valid data. The triangulation is then constructed inside this convex hull. This does not guarantee ipso facto a one-to-one correspondence between the boundaries of the convex hull and those of the area of interest (AOI). It is thus mandatory to make sure that the AOI is always surrounded by pixels labeled as valid, naturally or artificially, with phase values either imposed by a priori knowledge or computed by averaging and/or fitting the values of the AOI border. 


\subsection{Dynamic regime}

In a dynamic regime context, each pixel signal is analyzed temporally and obeys naturally to the two-beam equation (Eq. 1), with significant fluctuations of modulation and background intensities. An EMD-processed pixel signal has an appropriate shape for subsequent phase extraction (basically, its local mean value has been set to zero) but obviously still features some random fluctuation of modulation. The first mode of the decomposition can indeed be used to easily compute the modulation at each instant. The HT of this first mode leads directly to the phase extraction ${ }^{1}$. In the reliable areas, i.e. where the pixel modulation is higher than a chosen threshold, the phase will be obtained up to an additive constant. The computation of the discrete instantaneous frequency, IF, - temporal derivative of the phase - allows to get rid of this constant, leaving a meaningful exploitable result. IF values in the under-modulated intervals are discarded (see Fig. 4). Each pixel being independent, when we gather again the temporal sets to form frames, we obtain arrays of valid discrete IF sampled on a fluctuating non-uniform grid. An interpolation step has then to take place to obtain arrays of discrete IFs uniformly sampled. A discrete integration step yields eventually the phase map at each instant. The interpolation operation includes, as for the static case, the definition of a fixed convex hull, the DT computation and the cubic spline interpolation.

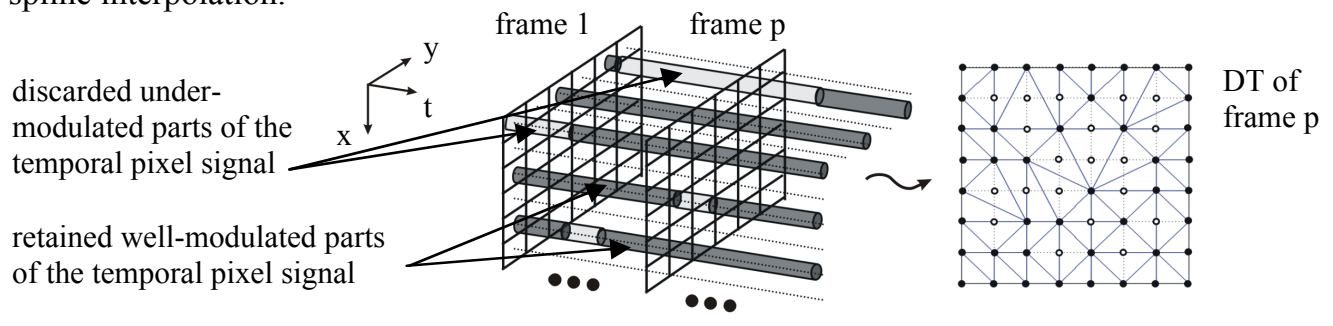

Figure 4: Data classification: (left) the irrelevant parts of the temporal signals are highlighted by bright tubes while the dark ones show the parts on which the interpolation step will rely; (right) the non-uniform sampling grid computed for an arbitrary frame with the associated DT.

The whole operation is illustrated in Fig. 5 with experimentally obtained signals in a Leendertz type speckle interferometer. The map of phase difference represents the in-plane displacement field of a continuously rotating diffusing metallic plate.

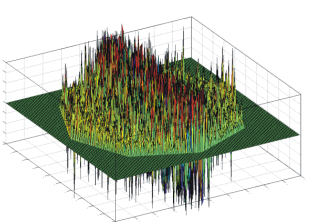

raw IF map at instant $\mathrm{k}$

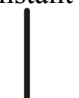

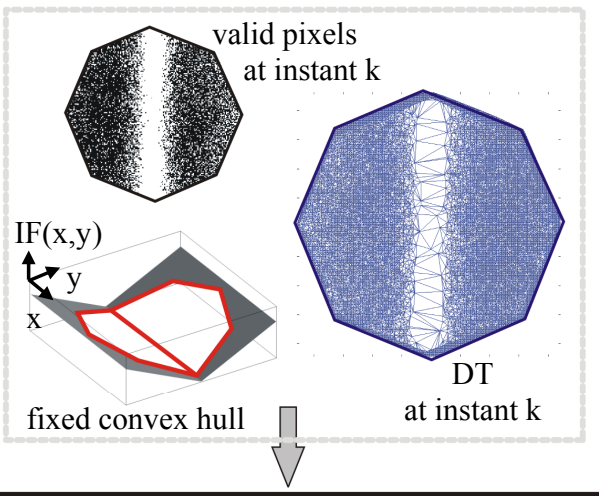

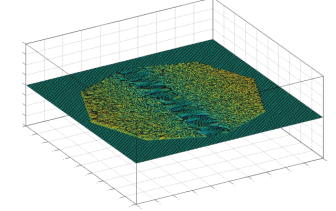

3DPP-processed IF map at instant $\mathrm{k}$

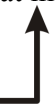

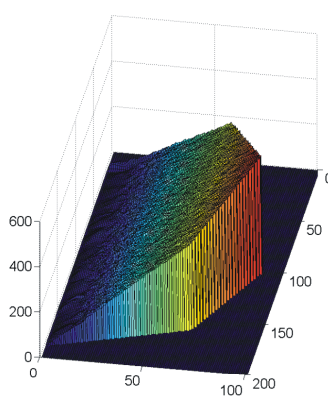

final phase map

Figure 5: From the definition of the fixed convex hull and the binary classification of the pixels at each instant, the DT is computed, and the 3DPP procedure yields a smoothed IF map where only the most reliable data have been kept. The final phase map is eventually obtained by summing the set of so-obtained IF maps.

In our Leendertz configuration, the line passing through the in-plane rotation axis on the object surface and parallel to the sensitivity vector is the locus of zero phase change. This a priori knowledge has been used for the definition of the fixed convex hull. It is a strong asset of the 3DPP method that the surrounding pixels on the convex hull can be assigned an IF value with a certain freedom, allowing for instance the preservation of sharp edges. 


\section{DT BASED FILTERING TECHNIQUES PROCESSING VERSUS OTHER FILTERING STRATEGIES}

\subsection{Spatial resolution}

Spatial resolution is usually defined by the minimum distance between independent measurements. Here, we suppose that the best achievable spatial resolution is merely the pixel itself. Depending on the threshold, the non-uniform sampling grid will be sparser, the tessellation will feature larger facets and the spatial resolution will then be correspondingly degraded. This is illustrated in Fig.6 with experimental signals obtained with an in-plane sensitive SI setup used to analyze the dynamic rigid body translation of a diffusing plate. The case where the threshold is chosen equal to the mean modulation intensity leaves $45 \%$ of the pixels as valid, while the case where the threshold is taken equal to twice the mean modulation intensity retains hardly $8 \%$ of the total amount of pixels. The length of the sides of the triangles is, in the first case, distributed between 1 and 4 pixels, and in the second case, is below 14 pixels; the mean side length values over the whole DT are respectively 1.9 and 6.8 pixels. Those values quantify the degradation of the spatial resolution at one instant.

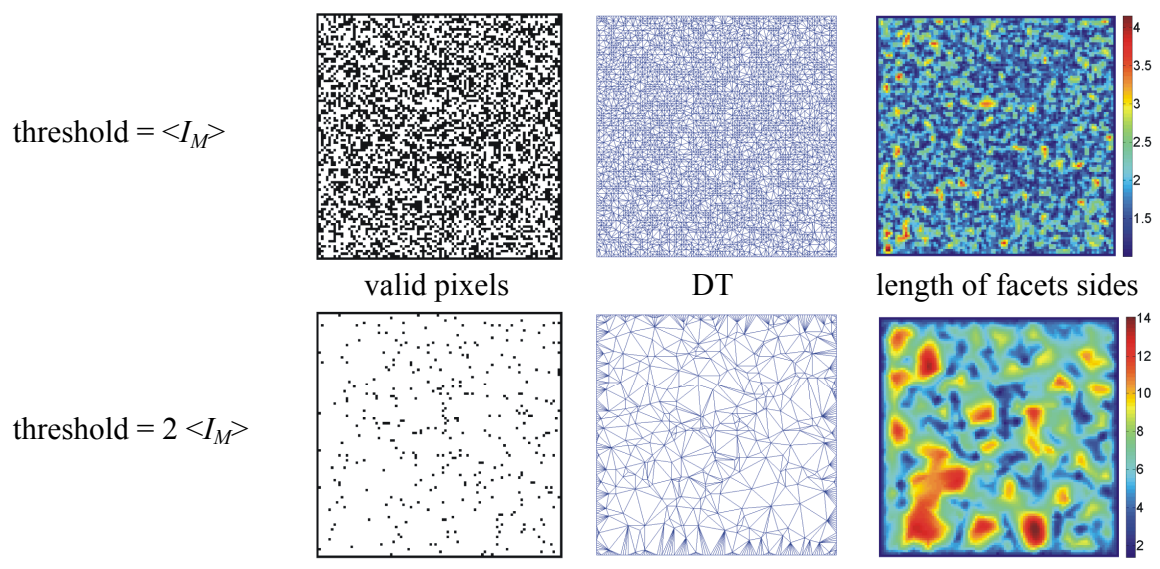

Figure 6: For two different values of the threshold, the maps of valid pixels are built and the corresponding DT is computed. The corresponding map of the mean side length (given in pixels) of each facet of the tessellation is also shown.

In static regime, some simulations have been carried out for assessing the performances of the sine-cosine DT filter. The mathematically defined reference phase signal $\Delta \varphi_{\text {th }}$ is merely a sinewave (Eq. 4) of fixed amplitude whose frequency is gradually increased. Such a phase definition might not be very representative of any physical behaviors (except maybe of a vibration mode) but it lends itself pretty well to a frequency analysis.

$$
\Delta \varphi_{\mathrm{th}}(x, y)=\frac{\pi}{2} \cos \left[\frac{2 \pi}{N} k x\right], \text { with } \quad k=1 \ldots N / 4
$$

The extracted phase, $\Delta \varphi_{\mathrm{ex}}$ obtained with a five frames phase-shifting algorithm from the simulated specklegrams [17] ${ }^{\dagger}$, is then filtered with five methods: $i$ ) the proposed sine-cosine DT filter, $i i)$ the basic sine-cosine filter with a box kernel whose size is given by the mean side length of the tessellation, iii) the sine-cosine filter with weights depending on the modulation with a kernel size also given by the mean side length of the DT, $i v)$ the sine-cosine filter with a $3 \times 3$ box kernel iterated 10 times and finally, $v$ ) the sine-cosine filter with modulation driven weights within a $3 \times 3$ kernel iterated 10 times. In each case, we compute the standard deviation of the difference between the filtered and the original phase, and the amplitude gain. The threshold used for the binary classification is a fraction of the mean modulation intensity $\left\langle I_{M}\right\rangle$ varying from $1 / 2$ to 1.25 . For visualization purpose and ease of analysis, the peak-valley of the sinewave which serves as the original phase is equal to $\pi$, i.e. less than one fringe. In this case, the frequency seen by the filter which acts upon the sine and the cosine of the phase will be simply the double of the original signal one.

\footnotetext{
$\dagger$ Simulated pairs of specklegrams (with smooth reference wave) obey Eq. (1) and are identical, except for the phase term $\Delta \varphi_{\text {th }}$ added to the specklegram representing the deformed state; the simulations have been carried out in a weak spatial integration regime $(m=2.5)$ in order to deal with the usual largest range of modulations; no decorrelations and no phase-shift errors are introduced, in order to isolate only the effects of low modulations.
} 
We report in Fig.7 the results of the simulations for different thresholds values. For each threshold, we computed the amplitude gain, i.e. the ratio between the amplitudes of the filtered phase and the original one, and the standard deviation of the phase difference still between the filtered and the original phases.
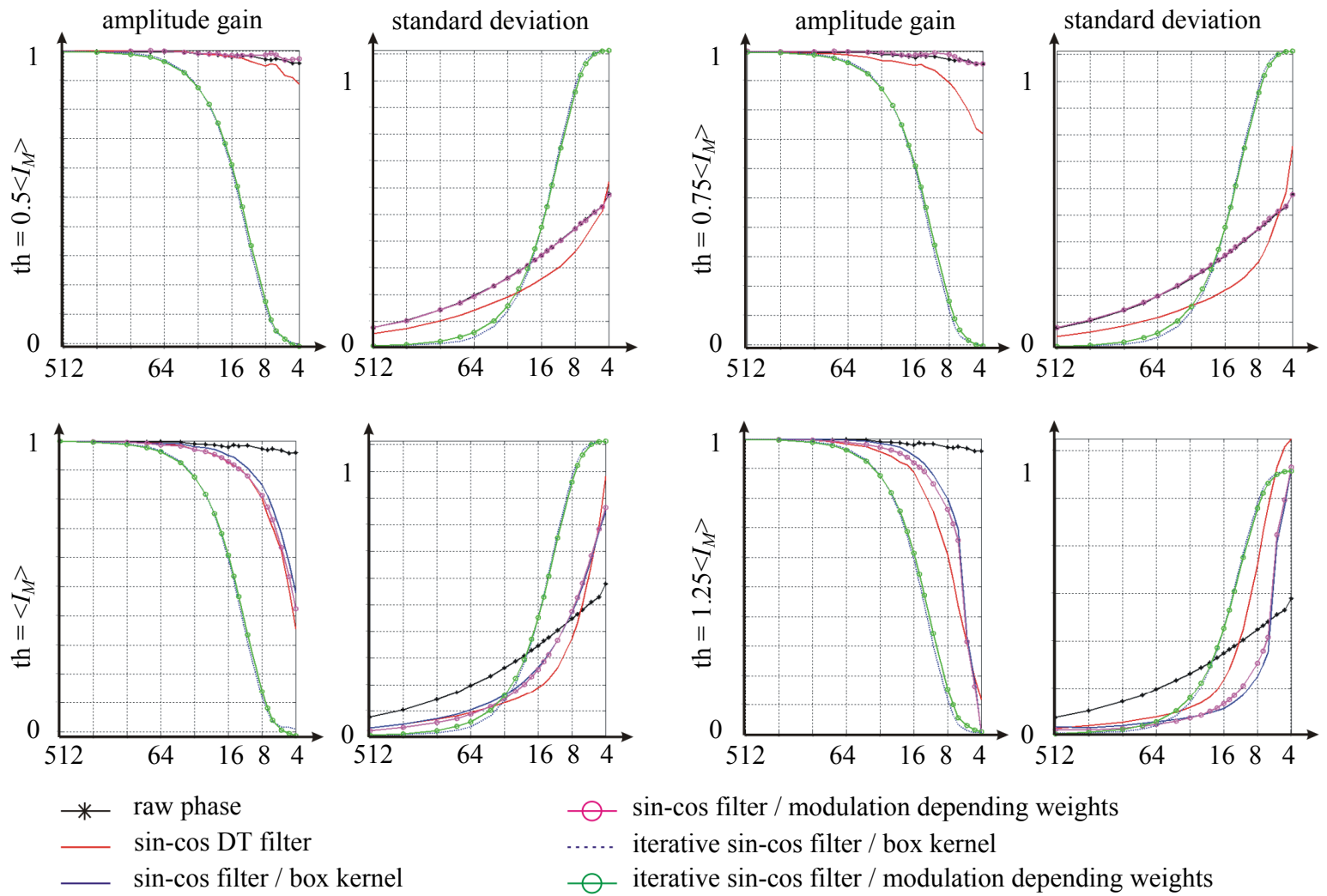

$\bigcirc$ sin-cos filter / modulation depending weights

...... iterative sin-cos filter / box kernel

$\bigcirc$ iterative sin-cos filter / modulation depending weights

Figure 7: For different values of the threshold (th $=0.5,0.75,1$ and $\left.1.25<I_{M}\right\rangle$ ), the preservation of the amplitude of the original sinewave (from 0 to 1 ) and the standard deviation between the outcome of the filtering procedure and still the original sinewave. The abscissa reports the number of samples per period (N/k in Eq. 4) of the sinewave in a log scale and ranges from 4 to $N=512$.

First of all, the overall spatial resolution on the phase map filtered with a $n \times n$ kernel iterative sine-cosine filter is not $n$ pixels. Indeed, the more the iterative sine-cosine filter is applied, the more the spatial resolution is degraded. It is moreover very interesting to note that for small thresholds, the sine-cosine DT filter decreases noticeably the noise while preserving the amplitude. It performs best than the classical sine-cosine filter since with a mean side length of the triangulation smaller than 2 (which is the encountered case when a small threshold is chosen), the filter is idle. For thresholds equal to or greater than the mean modulation intensities, it is wiser to use the classical sine-cosine filter. It turns out that the sine-cosine DT filter is an excellent tool to discard the most irrelevant pixels prior to the classical sinecosine filter application. It should allow to reduce the number of iterations for the same expectation in terms of phase smoothness and thus best preserve the spatial resolution.

It is worth mentioning that usually the spatial resolution is imposed by the filtering technique when a certain quality of the measurement is expected. Here, we rather fix a quality level, by imposing the modulation intensity threshold, and the spatial resolution is then automatically self-adapted. In addition, the classical conception of spatial resolution is restrictive for the dynamic regime case as it does not reflect the fact the non-uniform grid is fluctuating and a pixel which has been discarded at one instant can perfectly recover enough modulation to be taken as valid later on in the experiment. Even if the spatial resolution is definitely given locally by the size of the facet, the definition of the convex hull is very precious to best preserve the sharpness of edges, which cannot be guaranteed by classical filtering techniques with a kernel of fixed size. In Fig. 8, a typical phase map obtained in SI is given on the top left. A proper definition of the convex hull with constraints on the edges allows to build the DT within this fixed framework and reject noise in each sub-area without endangering the edges sharpness. 


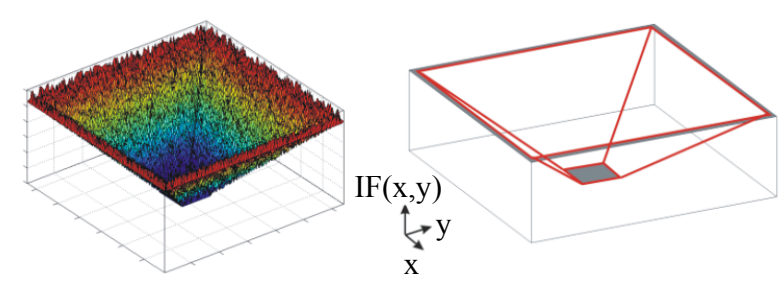

raw IF map

convex hull with constraints

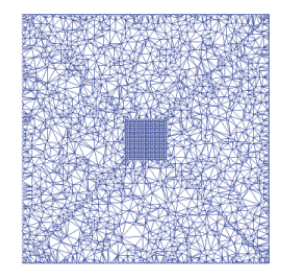

corresponding DT with a certain threshold

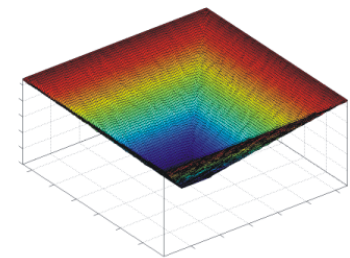

3DPP-processed IF map

Figure 8: A simulated example of convex hull with constraints to best preserve sharp edges.

\subsection{Algorithmic complexity}

The temporal approach needs to rearrange a 3D matrix (the two spatial coordinates of the sensor and the temporal coordinate) into a set of one-dimensional signals. Considering a matrix containing $N_{x} \times N_{y} \times N_{t}$ elements, the operation complexity is $O\left(N^{3}\right)$, even if the operation mainly involves memory allocation and is thus rather greedy in memory space than in processor resources. Moreover, a great amount of reading and writing operations can be saved if the $N_{x} \times N_{y}$ temporal signals are gathered in stacks ( $N_{x}$ stacks of $N_{y}$ signals for example).

At one instant $k$, the 3DPP technique is composed of three main steps. The first one is the binary classification of the data, which consists in a basic test over the $N_{x} \times N_{y}$ pixels. Given a certain threshold, the $N_{x} \times N_{y}$ values of modulation are compared to this threshold and a value 0 or 1 is then attributed to the pixel. This operation complexity is quadratic, i.e. $O\left(N^{2}\right)$. The DT is based on the $M$ valid points $\left(M \leq N_{x} \times N_{y}\right)$ and the Quickhull algorithm used to compute this tessellation is known to have a logarithmic complexity $O(M \cdot \log M)$. The last operation, the triangle-based interpolation, creates a smooth surface over the triangulation which can be further sampled on a regular grid. A trade-off must be found between noise removal, spatial resolution and computation load. A too low threshold will not discard regions of pixel signals with poor modulation intensity prone to the highest phase error. Conversely, a too high threshold will lead to a very discriminate test and the triangulation-interpolation step will rely on very few points, inducing a great loss of spatial resolution, with no significant improvement of the quality of the result. As an example, the processing of a batch of 1024 frames of $200 \times 200$ pixels lasts 20 minutes for a threshold which yields the best compromise between spatial resolution preserving and noise removing (49\% of discarded pixels), on a common computer equipped with a Core2 $2.66 \mathrm{GHz}$ processor.

\section{CONCLUSION}

In this paper, we propose a completely novel technique to cope with the under-modulation issue, intrinsically linked to SI signals, whatever static or dynamic regimes are in hands. The proposed methods show good behavior in low SNR regions. The sine-cosine DT filter constitutes a valuable pre-processing tool to discard the most aberrant pixels and then apply more classical filtering routines. In dynamic regime, and especially in long-range deformation measurements - i.e. for deformation range beyond the correlation length - the 3DPP technique yields excellent results and even allows to avoid carrier, when the direction of deformation or displacement is univocal, bearing in mind that the purpose of a carrier is to remove the phase sign ambiguity and to shift the rate of the deformation to a more suitable one for the acquisition system. Doing without carrier, again when the sign phase can be determined a priori, increases noticeably the bandwidth of measurement, and thus reduces substantially the required amount of data to characterize a given experiment.

Some improvements can be brought to be able to treat the case of objects with non-convex shapes, and the case of spatial discontinuities as well. Another point that would deserve to be pursued is the surface tiling to lighten the computation burden. A tiling depending on the activity with criteria derived from the temporal and spatial phase gradients would permit to sparsely select the pixels whose processing is the most significant (around a crack, in a high spatial displacement gradient area, ...).

\section{ACKNOWLEDGMENTS}

This work is supported by the Swiss National Science Foundation through the grant 200021_129489. 


\section{REFERENCES}

[1] Equis, S., and Jacquot, P., "The empirical mode decomposition: a must-have tool in speckle interferometry?" Optics Express 17, 611-623 (2009).

[2] Equis, S., and Jacquot, P., "A new application of the Delaunay triangulation: The processing of speckle interferometry signals", Fringe 2009, Springer, 123-131 (2009).

[3] Shvartsman, N., and Freund, I., "Speckle spots ride phase saddles sidesaddle", Opt. Comm., 117, 228-234 (1995).

[4] Ennos, A.E., "Speckle interferometry", [Progress in Optics XVI, E. Wolf ed.], North-Holland Publishing Company, Amsterdam, 233-288, (1978).

[5] Baranova, N.B., Zel'dovich, B.Y., Mamaev, A.V., Pilipetskii, N.F., and Shkunov, V.V., "Dislocations density on wavefront of a speckle-structure light field", Sov. Phys. JETP, 56, 983-988 (1982).

[6] Lehmann, M., "Statistical theory of two-wave speckle interferometry and its application to the optimization of deformation measurements", EPFL thesis n 1797, Lausanne, (1998).

[7] Lehmann, M., "Speckle statistics in the context of digital speckle interferometry", [Digital Speckle Pattern Interferometry and Related Techniques], John Wiley \& Sons, 1-58 (2001).

[8] Goodman, J.W., [Speckle phenomena in optics, theory and applications], Roberts and Company Publishers, Englewood, (2007).

[9] Goodman, J.W., "Statistical properties of laser speckle patterns", [Laser Speckle and Related Phenomena, J.C. Dainty ed.], $2^{\text {nd }}$ ed., Springer-Verlag, Berlin, (1984).

[10] Huntley, J.M., "Random phase measurements errors in digital speckle pattern interferometry", Opt. Laser Eng. 26, 131-150 (1997).

[11] Jacquot, P., "Speckle interferometry: a review of the principal methods in use for experimental mechanics applications", Strain, 44, 57-69 (2008).

[12] Aebischer, H.A., and Waldner, S., "Simple and effective method for filtering speckle interferometric phase fringe patterns", Opt. Comm., 162, 205-210 (1999).

[13] Equis, S., "Phase extraction of non-stationary signals produced in dynamic interferometry involving speckle waves", EPFL thesis $n^{\circ} 4514$, Lausanne, (2009).

[14] Bone, D.J., Bachor, H.A., and Sandeman, R.J., Fringe pattern analysis using a 2D Fourier transform, Appl. Opt., 25, 1653-1660 (1986).

[15] Colonna de Lega, X., "Processing of non-stationary interference patterns: adapted phase-shifting algorithms and wavelet analysis. Application to dynamic deformation measurements by holographic and speckle interferometry", EPFL thesis $n^{\circ} 1666$, Lausanne, (1997).

[16] Barber, C.B., Dobkin, D.P., and Huhdanpaa H., "The quickhull algorithm for convex hulls", ACM Transactions on Mathematical Software, 22(4), 469-83, (1996).

[17] Equis, S., and Jacquot, P., "Simulation of speckle complex amplitude: advocating the linear model", Proc. SPIE, Vol. 6341, 634138-1-6, (2006). 